\title{
Dynamics of angiogenesis and cellularity in rabbit VX2 tumors using contrast-enhanced magnetic resonance imaging and diffusion-weighted imaging
}

\author{
HAIXIA LI ${ }^{1}$, LIJUAN YU ${ }^{2}$, WENZHI WANG ${ }^{2}$, LINGLING WANG ${ }^{1}$, XIULAN ZHENG $^{1}$, \\ SHAOCHUN DAI ${ }^{1}$ and YANQIN SUN ${ }^{2}$ \\ Departments of ${ }^{1}$ Ultrasound and ${ }^{2}$ PET/CT, Harbin Medical University Cancer Hospital, \\ Harbin, Heilongjiang 150081, P.R. China
}

Received November 30, 2016; Accepted October 13, 2017

DOI: $10.3892 / \mathrm{ol} .2017 .7657$

\begin{abstract}
A number of studies have demonstrated that dynamic contrast-enhanced magnetic resonance imaging (DCE-MRI) may be used to evaluate microvessel density (MVD), and may quantitatively reflect tumor angiogenesis. To investigate the dynamics, including angiogenesis and tumor cellularity, of rabbit VX2 tumors during the 4 weeks following tumor implantation, the present study used DCE-MRI combined with diffusion-weighted imaging (DWI) to scan the tumors at 3 days, and then at 1,2, 3 and 4-week intervals, following tumor implantation. The dynamics, volume transfer coefficient $\left(\mathrm{K}^{\text {trans }}\right)$ and apparent diffusion coefficient (ADC) of the tumor parenchyma were analyzed. Furthermore, the associations between $\mathrm{K}^{\text {trans }}$ and MVD at 4 weeks after tumor implantation were analyzed. Tumor $\mathrm{K}^{\text {trans }}$ was positively correlated with MVD at 4 weeks $(\mathrm{r}=0.674, \mathrm{P}<0.001)$. Following tumor implantation, the tumor $\mathrm{K}^{\text {trans }}$ level rose for 2 weeks and then began to decline, reaching its lowest point at 4 weeks $(\mathrm{P}<0.001)$. ADC values at 1 week were higher than at 3 days, but declined thereafter $(\mathrm{P}<0.001)$. Tumor necrosis appeared by 1 week after tumor implantation. The necrosis degree of tumor was gradually increased from the occurrence of necrosis within the 4-week time span of the present study ( 1 vs. 2 weeks, $\mathrm{P}=0.008 ; 2$ vs. 3 weeks, $\mathrm{P}<0.001 ; 3$ vs. 4 weeks, $\mathrm{P}<0.001)$. The present study identified that tumor angiogenesis is a dynamic process that serves a function in tumor growth, and that DCE-MRI may reflect tumor parenchymal MVD and be useful in evaluating angiogenesis.
\end{abstract}

Correspondence to: Professor Lijuan Yu, Department of PET/CT, Harbin Medical University Cancer Hospital, 150 Ha Ping Road, Harbin, Heilongjiang 150081, P.R. China

E-mail: yulijuan0451@163.com

Key words: dynamic contrast-enhanced magnetic resonance imaging, diffusion-weighted imaging, tumor angiogenesis, VX2 tumor, rabbit, dynamic

\section{Introduction}

Tumor angiogenesis, which provides oxygen and nutrients to and removes metabolic waste from tumor cells, serves an important function in tumor cell proliferation and metastasis (1-3). An understanding of tumor angiogenesis is advantageous for evaluating the efficacy of tumor therapies, including radiation therapy, chemotherapy and interventional therapy $(4,5)$. Therefore, angiogenesis is a major focus of tumor research. Vascular endothelial growth factor (VEGF), which promotes a network of signaling processes that induce endothelial cell growth, migration and survival from preexisting vasculature and mediates vessel permeability, is a key mediator of tumor angiogenesis. It is also an anti-apoptotic factor for newly formed blood vessels and functions as an inducer of the mobilization of endothelial progenitor cells from bone marrow to distant sites (6,7). Microvessel density (MVD) has been demonstrated to be correlated with VEGF expression (8). As MVD increases, tumor aggressiveness also increases $(9,10)$. Currently, MVD is considered the 'gold standard' for measuring tumor angiogenesis (11).

It is important to measure angiogenesis longitudinally due to its dynamic nature (12). However, MVD measurement requires an invasive procedure, which, when coupled with specimen sampling error, causes limitations for its study (5). Additionally, in clinical practice there is a requisite to not only evaluate tumor angiogenesis, but also to obtain information regarding tumor morphology (e.g., tumor size, shape and the presence of necrosis) (13). For these reasons, a novel method for simultaneously obtaining data on angiogenesis and tumor morphology is required.

Due to recent developments in medical imaging, magnetic resonance imaging (MRI) may have a unique advantage for evaluating angiogenesis and tumor morphology. Dynamic contrast-enhanced (DCE)-MRI and its volume transfer coefficient $\left(\mathrm{K}^{\text {trans }}\right)$ have been demonstrated to correlate with MVD, and may quantitatively reflect tumor angiogenesis $(5,14)$. Furthermore, studies have demonstrated that diffusion-weighted imaging (DWI) and the apparent diffusion coefficient (ADC) are useful in evaluating cellularity and monitoring the tumor response to therapy $(15,16)$. 
In the present study, DCE-MRI combined with DWI and conventional MRI, including T1-weighted (T1W) and T2Wsequences, were used to investigate rabbit VX2 tumor changes during the 4 weeks following tumor implantation. Specifically, changes in angiogenesis, cellularity and necrosis were observed and recorded.

\section{Materials and methods}

Animals. The current study was approved by the Animal Research Committee of the Harbin Medical University Cancer Hospital (Harbin, China), and conducted in accordance with the guidelines of the International Council on Animal Care (17). A total of 15 male New Zealand white rabbits (3-4 months old and weighing $2.5-3.5 \mathrm{~kg}$, purchased from the Laboratory Animal Centre of the Harbin Medical University), were used in the present study. The rabbits were housed in individual cages under controlled laboratory conditions (temperature, $22 \pm 1^{\circ} \mathrm{C}$; air humidity, 50-60\%). Lights were on between 6:00 a.m. and 6:00 p.m., and animals had unrestricted access to food and water). The implanted VX2 tumor was originally grown in the right hind limb of two tumor-bearing rabbits. The tumors were removed from the muscles of tumor-bearing rabbit and cut into 1-2 $\mathrm{mm}^{3}$ blocks, which were placed in a small volume of normal saline, when the VX2 tumor grew to approximately $1-2 \mathrm{~cm}$ in diameter. Prior to tumor implantation, each rabbit was anesthetized with $2.5 \%$ pentobarbital sodium $(1 \mathrm{ml} / \mathrm{kg})$ via the auricular vein. Then the muscle tissue of the bilateral proximal shins of the hind-legs were cut open with the length of incision about $2.0 \mathrm{~cm}$. Next, either two or three VX2 tissue blocks were implanted into the muscular tissue of each of the bilateral proximal shins of the hind-legs. Finally, the muscle and skin were sutured.

Study protocol. MRI was performed 3 days after tumor implantation to confirm the successful establishment of rabbit VX2 models. MRI was repeated at 1,2, 3 and 4 weeks post-implantation. Following the final MRI scan, all rabbits were euthanized for histological analysis.

MRI. A clinical 3.0T MRI scanner (InteraAchieva; Philips Medical Systems, Inc., Bothell, WA, USA) with an 8-channel head coil was used. The MRI sequences included: i) T1W imaging: repetition time (TR)/echo time (TE), 543/20 msec; number of single averages (NSA), 6; slice thickness, $2 \mathrm{~mm}$; field of view (FOV), 100x100 mm; and matrix, 224x270; ii) T2W imaging: TR/TE, 1,600/100 msec; NSA,6; slice thickness, $2 \mathrm{~mm}$; FOV, 100x100 mm; and matrix, 200x192; iii) DWI: TR/TE, 1210/75 msec; NSA, 4; thickness, $3 \mathrm{~mm}$; FOV, 100x100 mm; and matrix, 92x90; iv) DCE-MRI; and v) post-T1W imaging. DWI was performed using a single-shot echo planar technique with $2 \mathrm{~b}$ values $\left(0,800 \mathrm{sec} / \mathrm{mm}^{2}\right)$ applied along 3 gradients, coinciding with 3 physical axes $(x, y$ and $z$ ). The 3D-THRIVE sequence (TR/TE=7.1/3.3 msec) with 2 and $15^{\circ}$ flip angles (FAs) were used to generate a T1-map. Next, 30 THRIVE measurements with $15^{\circ}$ FAs were used for DCE-MRI. Following construction of the T1-map and a first baseline measurement, $0.1 \mathrm{mmol} / \mathrm{kg}$ Gd-DTPA $(0.5 \mathrm{~mol} / \mathrm{l}$ Magnevist; Bayer HealthCare Pharmaceuticals, Guangzhou, Guangdong, China) was injected into the auricular vein at a constant rate of $0.2 \mathrm{ml} / \mathrm{kg} / \mathrm{min}$ with an automated injector (Sino Angio, Shenzhen, China). The temporal resolution of THRIVE was $13 \mathrm{sec}$.

Data processing. The DCE-MRI images were transferred to IDL-based image permeability processing software (Cine Tool version 6.6.1; GE Healthcare, Chicago, IL, USA). The femoral artery was selected as the input artery. The permeability parameter $\mathrm{K}^{\text {trans }}$, which represents the transport rate of the contrast agent from the intravascular space into the extravascular extracellular space (EES), was calculated based on the two-compartment modified Tofts model, which assumes that the total concentration of contrast agent in a tissue is equal to the concentrations in the two compartments, namely the intravascular and the extravascular extracellular spaces, and that no other compartments take up the contrast agent (18). The DWI images were transferred to a View Forum 5.1 workstation (Philips Medical Systems) to generate ADC maps. Post-T1W images, and the $\mathrm{K}^{\text {trans }}$ and ADC maps, were then transferred to 3DMed software (version 4.6; Medical Imaging Tool Kit, Beijing, China) for image registration prior to determination of the regions of interest (ROIs). ImageJ software (Version 1.47; National Institutes of Health, Bethesda, MD, USA) was used to manually draw the ROIs. Specifically, they were drawn along the parenchymal areas in the largest tumor slice, avoiding areas of necrosis by referring to the post-T1W images. Subsequently, the ROIs were copied and placed on the $\mathrm{K}^{\text {trans }}$ and ADC maps. Thigh muscle was used as a reference. In addition, the tumor volume was calculated using the following formula: [Area $\mathrm{x}$ slice number $\mathrm{x}$ (slice thickness + inter-slice gap)] based on the post-T1W images. Tumor necrosis was evaluated by using the necrosis degree (necrotic area/total area) in the largest slice determined based on the T1W, T2WI and post-T1W images.

Histological analysis. The MVD of the largest tumor slice was calculated under microscopy by marking the tumor vessel with a CD31 monoclonal antibody incubated for $1 \mathrm{~h}$ at room temperature (cat no., M082301; 1:50; Dako; Agilent Technologies, Inc., Santa Clara, CA, USA), according to Weidner's technique (19). Following the final MRI scan, rabbits were sacrificed by intravenous pentobarbital sodium overdose $(100 \mathrm{mg} / \mathrm{kg})$. Following fixation in $10 \%$ formaldehyde solution, the VX2 tumors located at the bilateral proximal shins and distal thighs of the hind-legs were removed in their entirety from the rabbits, embedded in paraffin and then sliced at $3 \mathrm{~mm}$ intervals in the axial plane, corresponding to the plane of the MR images. The section that corresponded to the largest slice of the tumor on the DCE-MRI was prepared for microscopic examination by routine immunohistochemical methods using the monoclonal mouse antibody against CD31 (Dako; Agilent Technologies, Inc.). Microvessels were counted as previously described (19). First, a low magnification (x40) was used to identify the areas of highest vascular density ('hot spots'), and then 3 'hot spots' were selected to count the number of microvessels under a high magnification (x200). The average value of these three counts represented the MVD for that case. A microvessel was considered as a brown-stained endothelial cell or an endothelial cell cluster with or without lumens which was clearly separated from adjacent tissue. Branches appeared with discrete breaks were also counted as single countable microvessels. 


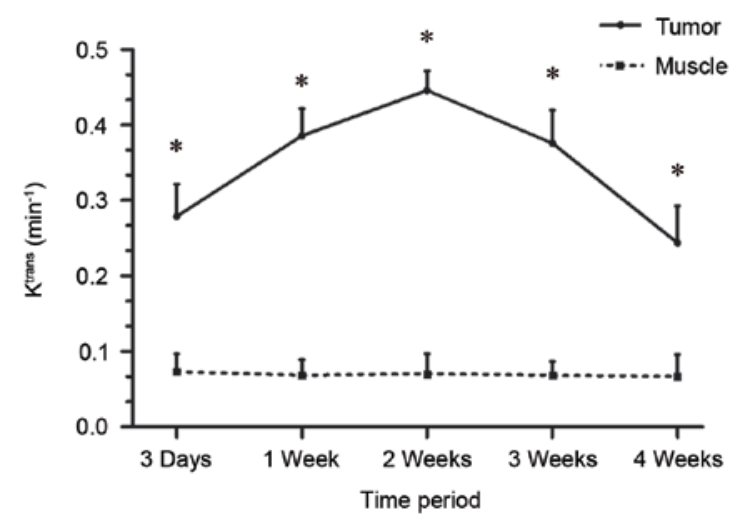

Figure 1 . Tumor $\mathrm{K}^{\text {trans }}$ level was higher than muscle $\mathrm{K}^{\text {trans }}$ level at each time point; ${ }^{\mathrm{P}}<0.001$. $\mathrm{K}^{\text {trans }}$, volume transfer coefficient.

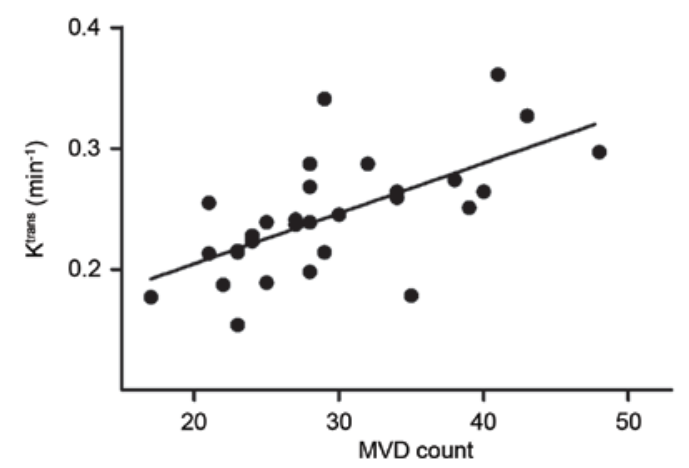

Figure 2. Tumor $\mathrm{K}^{\text {trans }}$ and MVD were positively correlated at 4 weeks; ${ }^{*} \mathrm{P}<0.001$. $\mathrm{K}^{\text {trans }}$, volume transfer coefficient; MVD, microvessel density.

Statistical analysis. SPSS software (version 16.0; SPSS, Inc., Chicago, IL, USA) was used for statistical analysis, and $\mathrm{P}<0.05$ was considered to indicate a statistically significant difference. Values are presented as the mean \pm standard deviation (SD). Differences between the tumor and muscle $\mathrm{K}^{\text {trans }}$ levels at 5 time points were calculated using the Mann-Whitney U test. Serial changes in $\mathrm{K}^{\text {trans }}$ and ADC across the five time points were compared using the Friedman test, followed by post hoc analysis with the Wilcoxon signed-rank tests with the Bonferroni correction. Correlations between tumor $\mathrm{K}^{\text {trans }}$ and MVD at 4 weeks, and between tumor $\mathrm{K}^{\text {trans }}$ and ADC at all time points, were calculated using the Spearman's correlation test. The necrosis degree of tumor (necrotic area/total area) was analyzed using the Friedman followed by post hoc analysis with Wilcoxon signed-rank tests with the Bonferroni correction.

\section{Results}

A total of $30 \mathrm{VX} 2$ tumors were present in the 15 experimental rabbits. The average tumor volume was $375.0 \pm 134.9$ at 3 days, and then $1,561.4 \pm 498.9,3,351.8 \pm 477.7,8,943.0 \pm 1,936.9$ and $1,8835.1 \pm 4,770.2 \mathrm{~mm}^{3}$ at $1,2,3$ and 4 weeks, respectively. Tumor growth was rapid, and necrosis appeared by 1 week after tumor implantation. The necrosis degree of tumor was gradually increased from the occurrence of necrosis with) in the 4-week time span of the present study ( 1 vs. 2 weeks, $\mathrm{P}=0.008 ; 2$ vs. 3 weeks, $\mathrm{P}<0.001 ; 3$ vs. 4 weeks, $\mathrm{P}<0.001$ ) (Table I). 

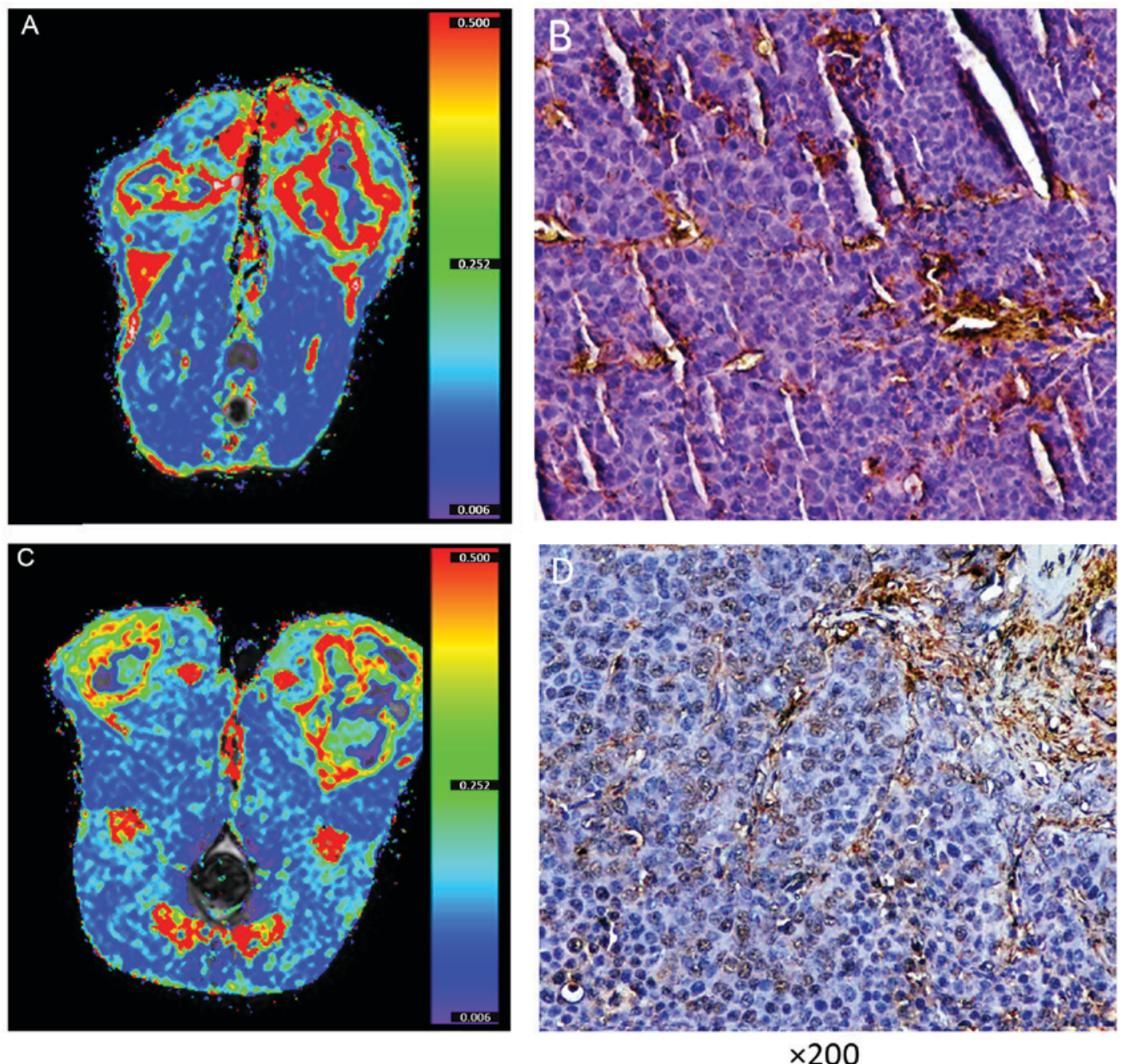

Figure 3. Two representativeVX2 rabbits. (A) Tumor $\mathrm{K}^{\text {trans }}$ map and (B) MVD was one rabbit and (C) $\mathrm{K}^{\text {trans }}$ map and (D) MVD was another rabbit. The MVD the of solid area of the VX2 tumor in one rabbit (B) was higher than the other rabbit (D), and the $\mathrm{K}^{\text {trans }}$ of the solid area of the VX2 tumor in one rabbit (A) was higher than the other rabbit (C) This suggests that the $\mathrm{K}^{\text {trans }}$ may reflect MVD and angiogenesis. $\mathrm{K}^{\text {trans }}$, volume transfer coefficient; MVD, microvessel density.

$K^{\text {trans }}$ measurements. No difference in the $\mathrm{K}^{\text {trans }}$ levels of the thigh muscles was identified amongst the 5 time points $\left(\mathrm{P}=0.430\right.$; Fig. 1), which indicated that the $\mathrm{K}^{\text {trans }}$ measurement was reliable in this study. Furthermore, the present study compared the tumor $\mathrm{K}^{\text {trans }}$ with MVD at 4 weeks and identified a positive correlation ( $\mathrm{r}=0.674, \mathrm{P}<0.001$; Figs. 2 and 3 ). This assisted in the confirmation that $\mathrm{K}^{\text {trans }}$ reflected MVD. Tumor $\mathrm{K}^{\text {trans }}$ was higher than muscle $\mathrm{K}^{\text {trans }}$ at each time point $\left(\mathrm{P}<0.001\right.$ for all). Furthermore, tumor $\mathrm{K}^{\text {trans }}$ values were significantly different between all adjacent time points $\left(\mathrm{P}<0.001\right.$ for all). Specifically, tumor $\mathrm{K}^{\text {trans }}$ values at 1 week were higher than those at 3 days, and those at 2 weeks were higher than at 1 week; tumor $\mathrm{K}^{\text {trans }}$ values at 3 weeks were lower than at 2 weeks, and those at 4 weeks were lower than at 3 weeks. Thus, the results demonstrate that, following tumor implantation, the tumor $\mathrm{K}^{\text {trans }}$ level rose for 2 weeks and then began to decline, reaching its lowest point at 4 weeks (Figs. 1, 4A and 5).

ADC measurements. The ADC values also differed between all adjacent time points $(\mathrm{P}<0.001$ for all). Specifically, the ADC values at 1 week were higher than at 3 days, but then declined consistently for up to 4 weeks. Cellularity was observed to reach its highest level 1 week following tumor implantation (Figs. 4B and 5).

Correlations between $K^{\text {trans }}$ and $A D C$. No correlation was revealed between the tumor $\mathrm{K}^{\text {trans }}$ and the ADC at any time point.

\section{Discussion}

In the present study, DCE-MRI and DWI, combined with conventional MRI, were used to monitor the VX2 tumor for a 4-week period following tumor implantation. The results suggest that the $\mathrm{K}^{\text {trans }}$ value quantitatively reflects tumor angiogenesis. Furthermore, angiogenesis of VX2 tumors was discovered to be a dynamic process that increased for 2 weeks following tumor implantation and began to decline thereafter. Changes in the tumor parenchyma were also identified, namely cellularity, and the extent of necrosis.

Previous studies have suggested that DCE-MRI reflects MVD and angiogenesis $(5,14)$. VX2 MVD at 4 weeks was used as a reference and $\mathrm{K}^{\text {trans }}$ was positively correlated with 

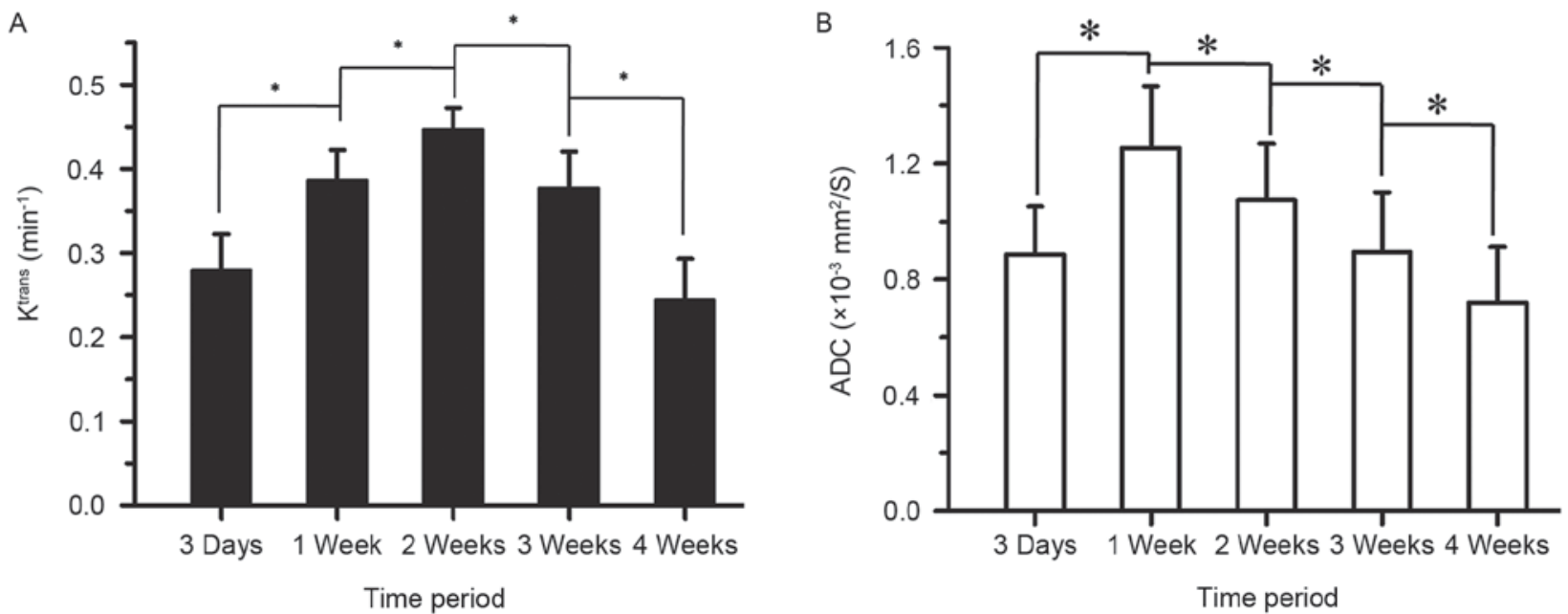

Figure 4. Changes in tumor (A) $\mathrm{K}^{\text {trans }}$ and (B) ADC over time following VX2 tumor implantation; (A) $\mathrm{K}^{\text {trans }}$ rose between 3 days and 2 weeks, then declined between 2 and 4 weeks; (B) ADC values increased between 3 days and 1 week, then decreased steadily from 1 to 4 weeks; ${ }^{*} \mathrm{P}<0.001$. $\mathrm{K}^{\text {trans }}$, volume transfer coefficient; ADC, apparent diffusion co-efficient.

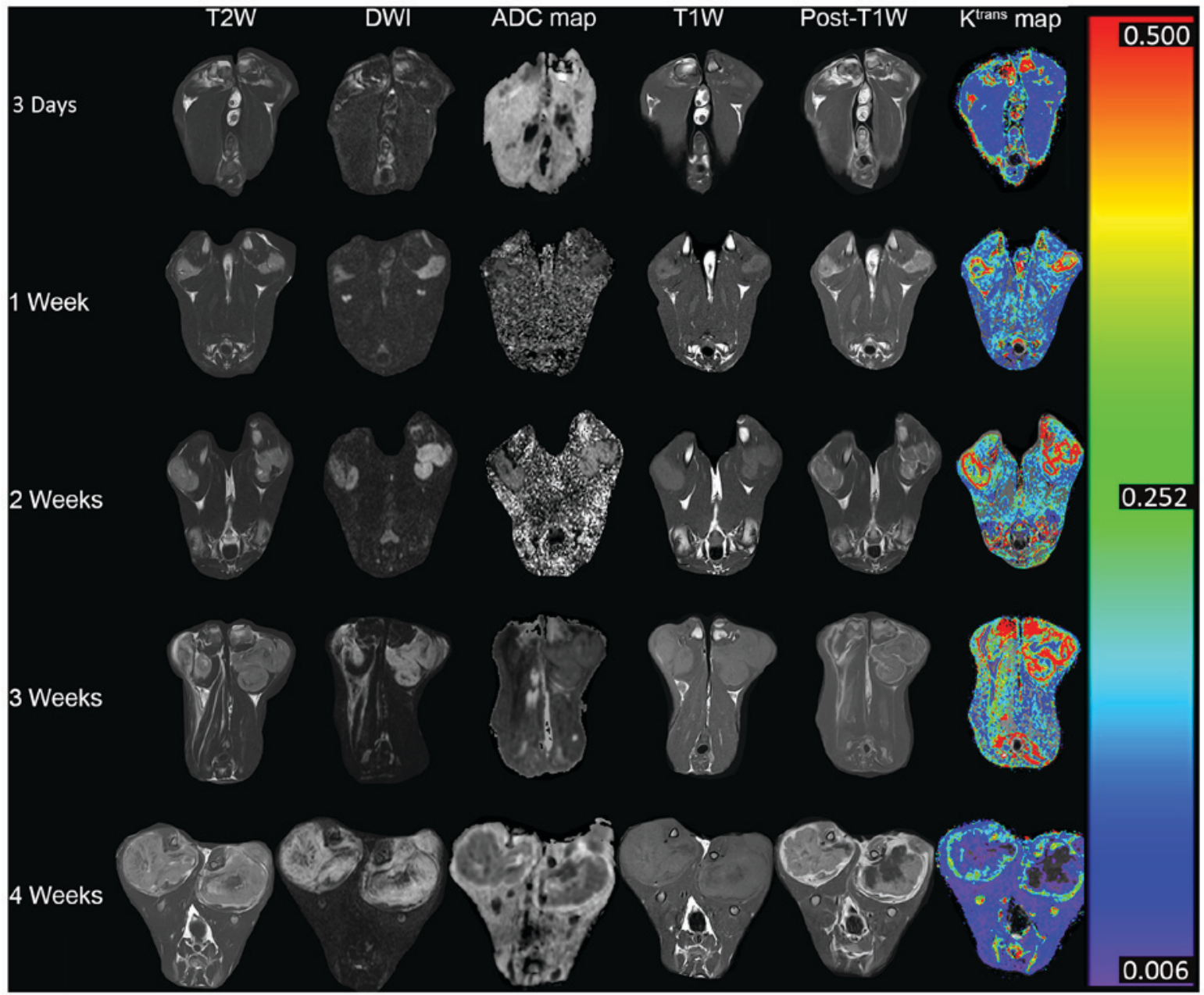

Figure 5. Magnetic resonance images from representative rabbits. At 4 weeks after tumor implantation, the tumor $\left(\mathrm{K}^{\text {trans }}\right.$ had decreased, and the rate and extent of necrosis had increased. $\mathrm{K}^{\text {trans }}$, volume transfer coefficient; TW1, T1-weighted; DWI, diffusion-weighted images.

MVD, indirectly reflecting tumor angiogenesis. It has been demonstrated that the neovasculature of malignant tumors lacks normal architecture, differing morphologically and hemodynamically from the vascular networks in normal tissues (10). These new, immature vessels have a high permeability (20). In the current study, the modified Tofts model was used to calculate $\mathrm{K}^{\text {trans }}$. This model assumes that the total amount of contrast agent in a tissue is determined 
by its concentration in the extravascular and intravascular extracellular spaces combined. $\mathrm{K}^{\text {trans }}$ represents the influx rate of contrast agent from the intravascular space to the extravascular extracellular space (18). Leakage of contrast agent into the extravascular extracellular space will increase with expansion of tumor angiogenesis. Therefore, $\mathrm{K}^{\text {trans }}$ may reflect VX2 tumor angiogenesis.

Understanding tumor angiogenesis and its dynamic process are of great value in the treatment of tumors. Although there have been numerous studies on angiogenesis in VX2 tumors $(21,22)$, the dynamic process of angiogenesis in VX2 tumors in the same animal, and the distinctive association between the dynamic processes of angiogenesis and of tumor growth require clarification. The present study demonstrated that VX2 tumor angiogenesis is a dynamic process, in that it increased for the initial 2 weeks after tumor implantation and then began to decline. Anti-angiogenic therapy has demonstrated efficacy (23) and thus, having an understanding of this dynamic process is useful.

Previous research has suggested that tumor growth requires neovasculature when the tumor diameter is $>2 \mathrm{~mm}$ (24). In the current study, the $\mathrm{K}^{\text {trans }}$ of the tumor parenchyma was higher than that of the muscle 3 days after tumor implantation, at which time tumor angiogenesis was evident. Tumor angiogenesis continued to rise until it peaked at 2 weeks. However, ADC values were higher at 1 week compared with at 3 days. It is recognized that the ADC value results from a combination of true molecular diffusion and microcirculatory perfusion $(25,26)$. Early on in tumor angiogenesis, the increase in ADC induced by microcirculatory perfusion may have been larger than the decrease in ADC from tumor cell propagation and proliferation. If so, it would provide an explanation as to why ADC values were higher at 1 week than at 3 days. Over time, tumor density and cellularity increased significantly, such that the ADC value began to decrease after 1 week.

The $\mathrm{K}^{\text {trans }}$ values of tumor tissues in the present study were higher than those of muscle tissues for each time point, indicating that tumor angiogenesis occurred throughout the entire study period. Although no correlation between $\mathrm{K}^{\text {trans }}$ and ADC values were identified for any time point, the ADC began to decrease after 1 week and continued to decline until 4 weeks. This demonstrates that tumor density and cellularity continued to rise, and suggests that tumor growth was accompanied by tumor angiogenesis throughout the study period. Absence of a correlation may be due to the small sample size, as well as a lack of synchronization between tumor angiogenesis and tumor cell propagation and proliferation.

Although the $\mathrm{K}^{\text {trans }}$ of the tumor parenchyma was higher than that of the muscle during the 4-week study time span, it began to decline at 2 weeks, reaching a minimum at 4 weeks, suggesting that tumor angiogenesis began to decrease at 2 weeks. Tumor necrosis was identified at 1 week, and the necrosis degree gradually increased with tumor development. Tumor necrosis is often associated with rapid growth and insufficient blood supply (27). Therefore, the increase in tumor angiogenesis after 2 weeks may be a reason for the higher rate and extent of necrosis. This also confirms the importance of tumor angiogenesis in tumor growth.

There are certain limitations to the present study. First, the present study did not analyze the correlation between $\mathrm{K}^{\text {trans }}$ and tumor size. Tumor size may be influenced by necrosis, which in turn, may induce bias in the evaluation of tumor angiogenesis and therefore, the association between $\mathrm{K}^{\text {trans }}$ and tumor size was not analyzed. Additionally, it is considered that the physiological indicators of the VX2 tumor are stable at 2 weeks following implantation. However, the $\mathrm{K}^{\text {trans }}$ and ADC continued to change 2 weeks after implantation in the current study. A previous study reported that certain diffusion MRI parameters (including ADC) may continue to change within the first month of tumor implantation (28). Therefore, the $\mathrm{K}^{\text {trans }}$ and ADC changes reflect the tumor angiogenesis and tumor celluarity, which may be the result of tumor physiological indicators and thus, may occur later than the changes in physiological indicators. Finally, the present study did not use other DCE-MRI parameters (e.g., Kep and Ve) to evaluate tumor angiogenesis. Kep (reverse reflux rate constant) is the reflux of the contrast agent from the EES to the intravascular space. Ve (volume fraction of EES) is the indirect measurement of the cellular density of the tissue (29). However, for Kep and Ve, there are some inconsistent results in evaluating tumor angiogenesis in different studies $(5,30,31) . \mathrm{K}^{\text {trans }}$, which represents the transport rate of the contrast agent from the intravascular space into the EES, may reflect tumor neovasculature more accurately and be used more widely $(5,32)$.

In conclusion, DCE-MRI and $\mathrm{K}^{\text {trans }}$, its quantitative coefficient, may reflect tumor parenchymal MVD and be helpful in evaluating angiogenesis. Thus, they may be useful tools for tumor management. Furthermore, tumor angiogenesis following VX2 implantation was identified to be a dynamic process, which continued to increase until reaching a peak at 2 weeks following tumor implantation, and then began to decrease; this process also appears to serve a function in tumor growth.

\section{Acknowledgements}

The present study was supported the National Natural Science Foundation of China (grant no. 81671771) and the grant of Harbin Science and Technology Bureau (grant no. 2016RAXYJ066).

\section{References}

1. Folkman J: Role of angiogenesis in tumor growth and metastasis. Semin Oncol 29 (6 Suppl 16): S15-S18, 2002.

2. Iagaru A and Gambhir SS: Imaging tumor angiogenesis: The road to clinical utility. AJR Am J Roentgenol 201: W183-W191, 2013.

3. Nomura T, Hirata K, Shimaoka T, Yamakawa M, Koizumi N, Suzuki R, Maruyama K and Utoguchi N: Cancer vaccine therapy using tumor endothelial cells as antigens suppresses solid tumor growth and metastasis. Biol Pharm Bull 40: 1661-1668, 2017.

4. Gerstner ER, Emblem KE and Sorensen GA: Vascular magnetic resonance imaging in brain tumors during antiangiogenic therapy-are we there yet? Cancer J 21: 337-342, 2015.

5. Chen J, Qian T, Zhang H, Wei C, Meng F and Yin H: Combining dynamic contrast enhanced magnetic resonance imaging and microvessel density to assess the angiogenesis after PEI in a rabbit VX2 liver tumor model. Magn Reson Imaging 34: 177-182, 2016.

6. Pinto MP, Owen GI, Retamal I and Garrido M: Angiogenesis inhibitors in early development for gastric cancer. Expert Opin Investig Drugs 26: 1007-1017, 2017.

7. Peng FW, Liu DK, Zhang QW, Xu YG and Shi L: VEGFR-2 inhibitors and the therapeutic applications thereof: A patent review (2012-2016). Expert Opin Ther Pat 27: 987-1004, 2017. 
8. Chen $\mathrm{HF}$ and $\mathrm{Wu} \mathrm{KJ}$ : Endothelial Trans differentiation of tumor cells triggered by the Twist1-Jagged1-KLF4 Axis: Relationship between Cancer Stemness and Angiogenesis. Stem Cells Int 2016: 6439864, 2016.

9. Bremnes RM, Camps C and Sirera R: Angiogenesis in non-small cell lung cancer: The prognostic impact of neoangiogenesis and the cytokines VEGF and bFGF in tumours and blood. Lung Cancer 51: 143-158, 2006.

10. Mischi M, Turco S, Lavini C, Kompatsiari K, de la Rosette JJ, Breeuwer $\mathrm{M}$ and Wijkstra $\mathrm{H}$ : Magnetic resonance dispersion imaging for localization of angiogenesis and cancer growth. Invest Radiol 49: 561-569, 2014.

11. Jia WR, Chai WM, Tang L, Wang Y, Fei XC, Han BS and Chen M: Three-dimensional contrast enhanced ultrasound score and dynamic contrast-enhanced magnetic resonance imaging score in evaluating breast tumor angiogenesis: Correlation with biological factors. Eur J Radiol 83: 1098-1105, 2014.

12. McCarville MB, Coleman JL, Guo J, Li Y, Li X, Honnoll PJ, Davidoff AM and Navid F: Use of quantitative dynamic Contrast-Enhanced ultrasound to assess response to antiangiogenic therapy in children and adolescents with solid malignancies: A pilot study. AJR Am J Roentgenol 206: 933-939, 2016.

13. Nedelcu RI, Zurac SA, Brînzea A, Cioplea MD, Turcu G, Popescu R, Popescu CM and Ion DA: Morphological features of melanocytic tumors with depigmented halo: Review of the literature and personal results. Rom J Morphol Embryol 56 (2 Suppl): S659-S663, 2015.

14. Arteaga-Marrero N, Rygh CB, Mainou-Gomez JF, Nylund K, Roehrich D, Heggdal J, Matulaniec P, Gilja OH, Reed RK, Svensson L, et al: Multimodal approach to assess tumour vasculature and potential treatment effect with DCE-US and DCE-MRI quantification in CWR22 prostate tumour xenografts Contrast Media Mol Imaging 10: 428-437, 2015.

15. Razek AA, Megahed AS, Denewer A, Motamed A, Tawfik A and Nada N: Role of diffusion-weighted magnetic resonance imaging in differentiation between the viable and necrotic parts of head and neck tumors. Acta Radiol 49: 364-370, 2008.

16. Ludwig JM, Camacho JC, Kokabi N, Xing M and Kim HS: The role of Diffusion-weighted imaging (DWI) in Locoregional therapy outcome prediction and response assessment for hepatocellular carcinoma (HCC): The New Era of Functional imaging biomarkers. Diagnostics (Basel) 5: 546-563, 2015.

17. International Council for Laboratory Animal Science (ICLAS). http://www.iclas.org. Accessed July 13, 2012.

18. Tofts PS, Brix G, Buckley DL, Evelhoch JL, Henderson E, Knopp MV, Larsson HB, Lee TY, Mayr NA, Parker GJ, et al: Estimating kinetic parameters from dynamic contrast-enhanced T(1)-weighted MRI of a diffusable tracer: Standardized quantities and symbols. J Magn Reson Imaging 10: 223-232, 1999.

19. Weidner N: Current pathologic methods for measuring intratumoral microvessel density within breast carcinoma and other solid tumors. Breast Cancer Res Treat 36: 169-180, 1995.

20. Hida K, Hida Y and Shindoh M: Understanding tumor endothelial cell abnormalities to develop ideal anti-angiogenic therapies. Cancer Sci 99: 459-466, 2008.
21. Lijowski M, Caruthers S, Hu G, Zhang H, Scott MJ, Williams T, Erpelding T, Schmieder AH, Kiefer G, Gulyas G, et al: High sensitivity: High-resolution SPECT-CT/MR molecular imaging of angiogenesis in the Vx2 model. Invest Radiol 44: 15-22, 2009.

22. Feng G, Lei Z, Wang D, Xu N, Wei Q, Li D and Liu J: The evaluation of anti-angiogenic effects of Endostar on rabbit VX2 portal vein tumor thrombus using perfusion MSCT. Cancer Imaging 14: 17, 2014.

23. Manzo A, Montanino A, Carillio G, Costanzo R, Sandomenico C, Normanno N, Piccirillo MC, Daniele G, Perrone F, Rocco G and Morabito A: Angiogenesis Inhibitors in NSCLC. Int J Mol Sci 18: pii: E2021, 2017.

24. Folkman J: What is the evidence that tumors are angiogenesis dependent. J Natl Cancer Inst 82: 4-6, 1990.

25. Thoeny HC, De Keyzer F, Vandecaveye V, Chen F, Sun X, Bosmans H, Hermans R, Verbeken EK, Boesch C, Marchal G, et al: Effect of vascular targeting agent in rat tumor model: Dynamic contrast-enhanced versus diffusion-weighted MR imaging. Radiology 237: 492-499, 2005.

26. Panagiotaki E, Walker-Samuel S, Siow B, Johnson SP, Rajkumar V, Pedley RB, Lythgoe MF and Alexander DC: Noninvasive quantification of solid tumor microstructure using VERDICT MRI. Cancer Res 74: 1902-1912, 2014.

27. Chen FH, Wang CC, Liu HL, Fu SY, Yu CF, Chang C, Chiang CS and Hong JH: Decline of tumor vascular function as assessed by dynamic Contrast-Enhanced magnetic resonance imaging is associated with poor responses to radiation therapy and chemotherapy. Int J Radiat Oncol Biol Phys 95: 1495-1503, 2016.

28. Wu H, Liu H, Liang C, Zhang S, Liu Z, Liu C, Liu Y, Hu M, $\mathrm{Li} \mathrm{C}$ and Mei Y: Diffusion-weighted multiparametric MRI for monitoring longitudinal changes of parameters in rabbit VX2 liver tumors. J Magn Reson Imaging 44: 707-714, 2016.

29. Chen BB and Shih TT: DCE-MRI in hepatocellular carcinoma-clinical and therapeutic image biomarker. World J Gastroenterol 20: 3125-3134, 2014

30. Kim SH, Lee HS, Kang BJ, Song BJ, Kim HB, Lee H, Jin MS and Lee A: Dynamic Contrast-Enhanced MRI perfusion parameters as imaging biomarkers of angiogenesis. PLoS One 11: e0168632, 2016.

31. Kim YE, Lim JS, Choi J, Kim D, Myoung S, Kim MJ and Kim KW: Perfusion parameters of dynamic Contrast-enhanced magnetic resonance imaging in patients with rectal cancer: Correlation with microvascular density and vascular endothelial growth factor expression. Korean J Radiol 14: 878-885, 2013.

32. Zhang W, Chen HJ, Wang ZJ, Huang W and Zhang LJ: Dynamic contrast enhanced MR imaging for evaluation of angiogenesis of hepatocellular nodules in liver cirrhosis in N-nitrosodiethylamine induced rat model. Eur Radiol 27: 2086-2094, 2017.

This work is licensed under a Creative Commons Attribution-NonCommercial-NoDerivatives 4.0 International (CC BY-NC-ND 4.0) License. 